\title{
Design of Energy-saving Fluorescent Lamp System
}

\author{
Chunyan Xie \\ College of Information Technology Hebei Normal University, Shijiazhuang, China \\ xiecynu@163.com
}

Keywords: Energy-saving, STC, Fluorescent Lamp, circuit

\begin{abstract}
To solve the problem of energy waste caused by fixed luminance of common lamps, a new fluorescent lamp system is designed which is based on STC SCM. In this system, Light gathering is used to collect and analyzes the light luminance outside by STC; the circuit composed by the PWM is controlled the fluorescent light intensity, which can automatic adjust light luminance according to different environment. It reduces greatly the power of fluorescent lamp and energy waste, and could be applied widely.
\end{abstract}

\section{Introduction}

With the development of the society and the growth of population, energy saving has become an important social issue. The common illuminant which is used widely always keeps the same luminance no matter how bright the environment is, so huge energy wastes are caused. In this paper a new illuminant system is proposed based on STC single chip microcomputer (SCM). By gathering information of light luminance outside, SCM STC12 changes light luminance of the illuminant with the environment automatically to save energy. This system has simple structure, high reliability and low cost and could be used widely in school and family life.

\section{The Circuit of the Hardware Composition}

The hardware structure of the system. System structure is as shown in figure 1 . The light gathering part is mainly composed of photosensitive resistance, triode and its peripheral circuit. SCM controller part mainly consists of single-chip computer STC12 and its peripheral circuit analogue-to-digital converter ADC0809. Automatic adjustment part o is mainly composed of optical coupling MOC3052, bidirectional triode BT136 and its peripheral circuit. The light gathering part senses the light luminance outside by photosensitive resistance, and changes its resistance with the light luminance outside to change its output voltage. SCM controller converts the analog voltage to the digital quantity by ADC0809, and puts out duty cycle controlled PWM wave after analysis. Automatic adjustment part of the system regulates and controls the voltage at the ends of the lamp tube according to the wave PWM, and it could achieve a high accuracy about $0.1 \mathrm{~V}$ to control the irradiation luminance.

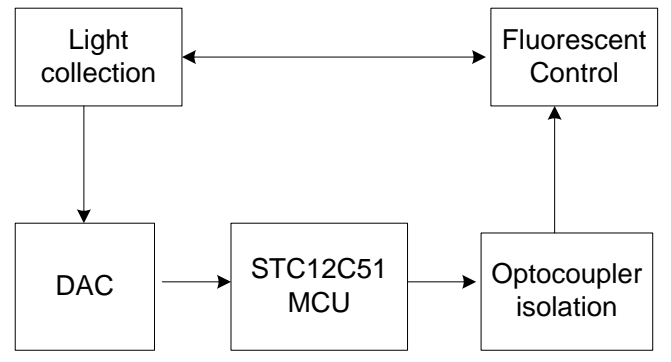

Fig. 1 System hardware structure

Circuit of Light Gathering. As shown in figure 2, the design adopts emitter bias circuit on base divider, and is composed of Vcc (5 V), base resistance R5, R1 and the collector resistance R6 and its triode emitter grounds directly. R5 and R6 are ordinary resistance, and R1 is photosensitive resistance. The circuit has good stability, and resistance R5 pick up directly on the transistor's base to control the base current, so it can prevent effectively resistance tolerance fluctuations which is caused by the temperature influence and other factors. Photosensitive resistance senses the light 
luminance outside directly, whose spectrum peak could reach $540 \mathrm{~nm}$, light resistance (lux10) 5 $10 \mathrm{k} \Omega$, and dark resistance $0.6 \mathrm{M} \Omega$.

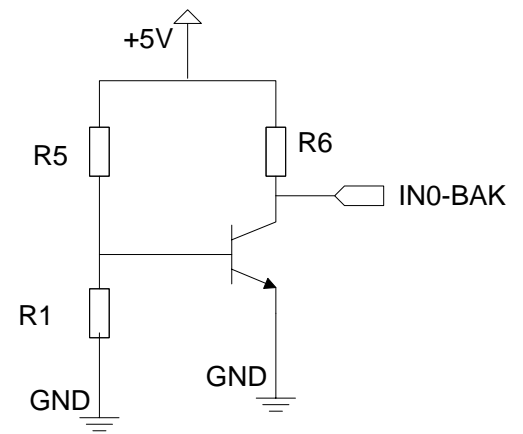

Fig. 2 Light intensity acquisition circuit

When the light luminance gets stronger, photosensitive resistance R1 increases, current IR1 gets smaller, and triode base current Ib decreases, so the collector voltage, that is the output voltage IN0-BAK, gets bigger. On the contrary, when the light luminance gets weaker, output voltage INO-BAK gets smaller. So triode will transform the current changes caused by light luminance changes into output voltage changes, which is put in port access of ADC0809.

The Design of A/D Convertor Circuit. This design adopts 8-bit ADC0809. The chip is a typical 8-bit successive approximation A/D converter with 8 channels, and can realize 8 analog voltage conversions. For simple conversion, its reference voltage is set at $5 \mathrm{~V}$, that means the analog input is + 5.0, the output of ADC is $0 \mathrm{FFH}$, that is 225, system resolution is 50/255 = 0.2 V/LSB. Reference voltage is provided by the precision voltage regulator LM7805.LM7805 is connected as shown in figure 3, J1 is supplied with dc $12 \mathrm{~V}$ power, C6 is input filter capacitor and C7 is output one, C12 is input resistance, and C3 is load resistance.

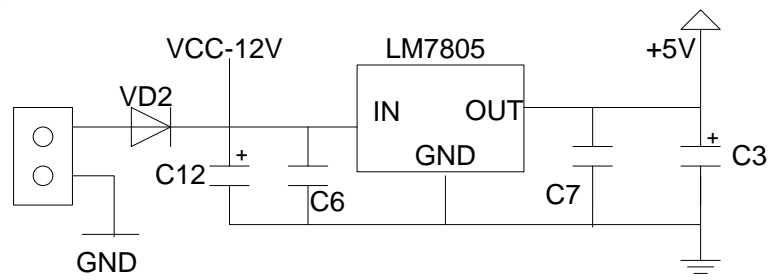

Fig. 3 LM7805 Regulator circuit

Automatic Adjustment Circuit. As shown in figure 4, this module is the core in the circuit. $\mathrm{P} 1.3$ is the input of PWM wave, high electrical level is $5 \mathrm{~V}$ and the low one is $0 \mathrm{~V}$. R11 is a pull-up resistor to limit current. Photoelectric coupler MOC3052, which builds effective isolation between low voltage circuit and high voltage circuit, is used as component SMD. J2 is supplied with $220 \mathrm{~V}$ ac power, J3 grounds and fluorescent lamp resistance R10 connects with live wire and ground wire. R8 and R7 are the Symmetric high-power resistance for the use of current limiting.

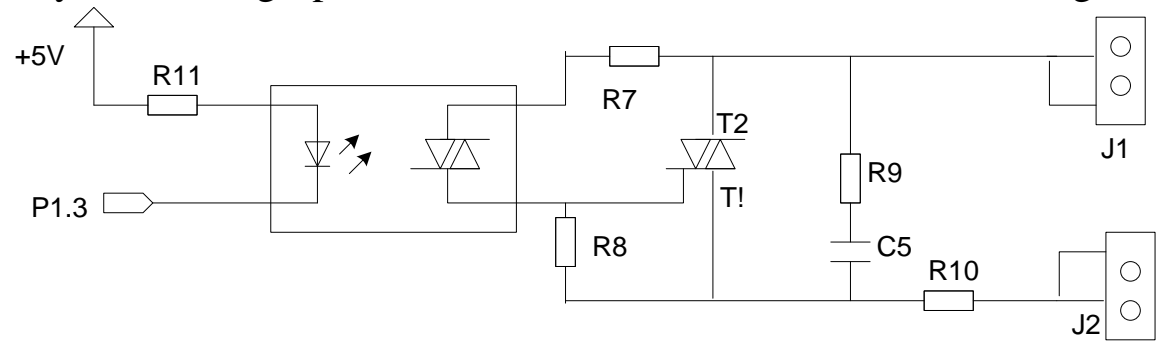

Fig. 4 Fluorescent lamp control circuit

\section{The Design of Software}

The main task of the system is to monitor the luminance outside in real time, and then send PWM wave through SCM through to control the opening and closing of light coupling to control the luminance of fluorescent lamp. The software of the system focuses on SCM programming. it is as shown in figure 5. 


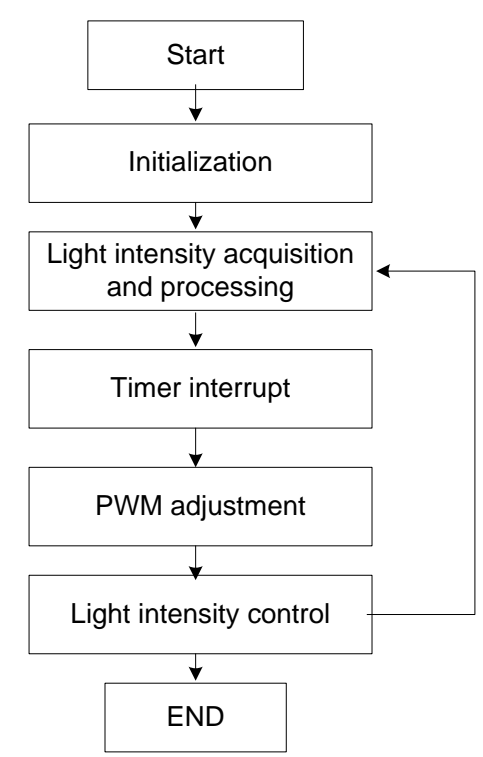

Fig. 5 The main program flow chart

\section{The Test of the System}

An independent illuminant whose luminance could be variable is used to shine the photosensitive resistance (about $20 \mathrm{~cm}$ away). Light luminance changes from dark to light gradually, and then test the voltage at both ends of the fluorescent lamp. A photometer is placed at the distance of $1 \mathrm{~m}$ from the tubes to test light luminance, and a power meter neat the tube to test power. In the process of the test, the photosensitive resistance only receives the independent illuminant, and the photometer only receives light of fluorescent lamp. The light luminance of fluorescent lamp varies is on the range of 170 to $245 \mathrm{~V}$, and do the test 10 times. The results are as shown in table 1 . in the table data of light luminance for spectrophotometer is the average of data measured 10 times, and power ratio is the value of the average power value of 10 times measured divided by power of the fluorescent lamp (40 W) as shows at The table 1 , it could be concluded that the new type of fluorescent lamp could save energy effectively with enough luminance. After debugged repeated, it is sensitive to the light luminance, and it has a larger luminous range with stable light. It is suitable for daily life.

Tab. 1 Experimental Data

\begin{tabular}{ccc}
\hline Voltage/V & Light intensity/LUX*10 & Power Ratio/\% \\
\hline 243.0 & 485 & 99.2 \\
\hline 240.0 & 463 & 96.1 \\
\hline 236.0 & 446 & 92.2 \\
\hline 232.0 & 421 & 88.4 \\
\hline 227.0 & 396 & 84.3 \\
\hline 220.0 & 384 & 81.1 \\
\hline 212.0 & 358 & 77.4 \\
\hline 210.0 & 314 & 74.6 \\
\hline 204.0 & 312 & 69.7 \\
\hline 200.0 & 290 & 67.1 \\
\hline
\end{tabular}

\section{Conclusion}

In this paper, a new fluorescent illuminant system is designed which is based on single chip microcomputer to solve the problem of energy waste caused by fixed light luminance of common lamps. This system collects and analyzes the light luminance outside, and adjusts light luminance of fluorescent lamp by the SCM according to different environment. It reduces greatly the power of fluorescent lamp and energy waste, and should be promoted widely. 


\section{References}

[1] J.H. Wang, Laser processing technology enables solar cells with $22 \%$ coversion efficiency. OME Information, 24 (7):23-24(2010).

[2] C.Y. He, Solar-based STC12C5410AD lighting controller. Electronic Design Engineering.3:12-13(2011)

[3] F.C. Xu, Energy saving control system of campus lighting based on LED. ModernElectronicsTechnique. vol 37. (5):156-162(2014)

[4] G.W. Li, The advantages of Led energy-saving lamps and the design concept. Popular Science\&Technology.Vol 15.(5):126-129(2013)

[5] Y.Y. Zhang, The exploration of LED tube design. Hunan Agricultural Machinery. (1): 102-105(2013)

[6] K. Zhang, Design of energy-efficient lighting control system based on single chip microcontroller. Hebei Journal of Industrial Science and Technology. Vol.28(2):104-107(2011)

[7] Z.D. Xie, Design of Energy Economizer of Intelligent Street Lamp Based on P89C51RD2 Microcontroller Control. Microcomputer Information.Vol.21(3):80-83(2011) 